\title{
Wireless Proximity Sensor in LoRaWAN Network
}

\author{
Michal Mihálik \\ University of Žilina, \\ Faculty of Electrical Engineering and \\ Information Technology, Department \\ of Control and Information Systems \\ Žilina, Slovak Republic \\ michal.mihalik@feit.uniza.sk
}

\author{
Marián Hruboš \\ University of Žilina, \\ Faculty of Electrical Engineering and \\ Information Technology, Department \\ of Control and Information Systems \\ Žilina, Slovak Republic \\ marian.hrubos@feit.uniza.sk
}

\author{
Emília Bubeníková \\ University of Žilina, \\ Faculty of Electrical Engineering and \\ Information Technology, Department \\ of Control and Information Systems \\ Žilina, Slovak Republic \\ emilia.bubenikova@feit.uniza.sk
}

\begin{abstract}
The aim of this work is to create a functional prototype of a wireless proximity sensor, which will send processed information via a wireless sensor network. The combination of wireless sensor network and low power devices create a network known as the LowPower Wide-Area Network (LPWAN). In this article, we will focus on the hardware configuration of the terminal and its testing.
\end{abstract}

Keywords-LoRaWAN; LPWAN; proximity sensor; ATMEGA328P-AN

\section{INTRODUCTION}

The Industry 4.0 Industry revolution is underway. This revolution clearly affects the development of new technologies. Companies and businesses that want to remain competitive in the industry, are striving to incorporate Industry 4.0 elements and solutions. The Industry 4.0 concept is inherently connected to the collection and evaluation of data from all available sources. Industrial applications in various fields are used for sensing and data collection different types of sensors [1], [2], [3]. Therefore, there are increased demands on computer technology, scanning technology, data storage, etc.

The result of this work should be a functional prototype proximity sensor, which will be connected to the wireless network. Networks that connect sensors are also called Wireless Sensor Networks (WSN). In many applications, WSN communicates with a Local Area Network (LAN) or a Wide Area Network (WAN) through a gateway. The gateway serves, as a bridge between WSN and another network. This allows data to be stored and processed by multiple resource devices, such as on a remote server. The well-known technology with which we can create the network WSN such as Bluetooth, ZigBee, Wi-Fi.

\section{WIRELESS SENSOR REQUIREMENTS}

As a first step, it was necessary to specify the requirements. Based on the requirements we have chosen appropriate technology. The requirements can be seen in Tab. 1
TABLE I. SENSOR REQUIREMENTS

\begin{tabular}{|c|l|}
\hline $\begin{array}{c}\text { Requirement } \\
\text { number }\end{array}$ & \multicolumn{1}{c|}{ Description of the requirement } \\
\hline 1. & Sensing material flow continuously for 2 weeks \\
\hline 2. & Counting the pieces that enter the line machine \\
\hline 3. & $\begin{array}{l}\text { Counting the pieces that getting off the line } \\
\text { machine }\end{array}$ \\
\hline 4. & Data transmission via wireless media \\
\hline 5. & Mid-range data transmission in production halls \\
\hline 6. & The power supply must be easily replaceable \\
\hline 7. & Contactless sensing of steel material \\
\hline 8. & Sensing range 0 -10 mm \\
\hline 9. & Communication does not have to be in real time \\
\hline
\end{tabular}

Based on the set requirements, it was necessary to select the technology that works in the Industrial Scientific Medical (ISM) frequency band. In the EU, this band is at 433; 868 and $2450 \mathrm{MHz}$. When we were choosing the appropriate technology, Wi-Fi has been rejected because it is often used in the industry. When we reviewed all the requirements, we chose technology Long Range (LoRa). LoRa communicates on the frequency band $868 \mathrm{MHz}$ and is variation Chirp Spread Spectrum (CSS) modulation. CSS modulation using the spread spectrum method. Spectrum spreading is typically used to increase interference immunity and increase the sensitivity of a signal receiver [4].

\section{LORAWAN AND LORA MODULATION}

The LoRaWAN is a Low Power Wide Area Network (LP-WAN) protocol and system architecture developed by LoRa Alliance while LoRa defines the physical layer of the system. In addition, LoRaWAN is a media access control (MAC) layer protocol built on top of the LoRa physical layer. Network architecture LoRa operates in a nonlicensed band below $1 \mathrm{GHz}$ [5]. The transmission channel of this technology is defined using three main parameters of the spreading factor (SF), bandwidth and carrier frequency. In this case, only the bandwidth value is 
constant. It is $125 \mathrm{kHz}$. Carrier frequency has a range of around $868 \mathrm{MHz}$. SF is used to adjust the degree of modulation. The SF will vary during the tests, based on the transmission property. This parameter can also be changed during operation by the device itself. The device changes SF if the message cannot be received for the second time. The change of SF is directly related to the range of the radio transmission, the transmission rate, but also the size of the transmitted message. Its values range from SF7 to SF12. We can see influence change SF on selected properties in Fig. $1[4]$. By setting these parameters, they are discussed in the source [6].

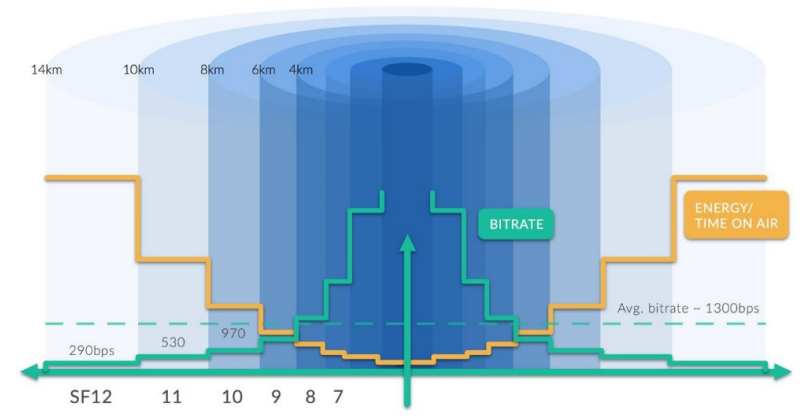

Figure 1. Transmission properties [4]

The limitation that increases with the use of this technology is called the Duty cycle. This parameter determines the transmission ratio. In the ISM band is the maximum value for this ratio $1 \%$. This means that in 1 hour the device can transmit 36 seconds. We will have to be considered this parameter in software implementation. As most effectively adapt the duty cycle analyzes source [7]. The basic LoRaWAN network architecture can be seen in Fig.2 [4].

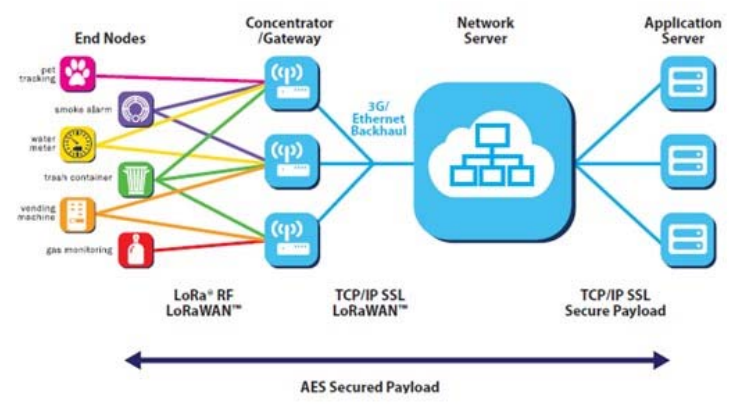

Figure 2. LoRaWAN network architecture. [4]

It consists of four parts application server, network server, gateway, and end node. In our case, it is represented by three devices. Application server and network server is running on one device specifically on the notebook. Gateway works on the Raspberry $\mathrm{Pi}$ platform, which is added module IC880A which supports LoRa modulation. The last part is the end node, and this is the wireless proximity sensor. LoRa modulation is used only between the end node and the gateway. Another communication is done via the IP network. The more detailed course of communication and security mechanisms in communication by LoraWan Protocol, are analyze the source [8] and [9]. How to configure the gateway can be found in the source[11]. On the gateway configuration can be used more options. We decided to use Linux operating system. Also, the application and network server use the Linux operating system.

\section{DESIGN SENSOR ARCHITECTURE}

When we were designing the architecture, we proceeded from the block diagram of Fig. 2, by means of which the issue was divided into five blocks. Based on Fig. 3, we had an issue composed of five parts.

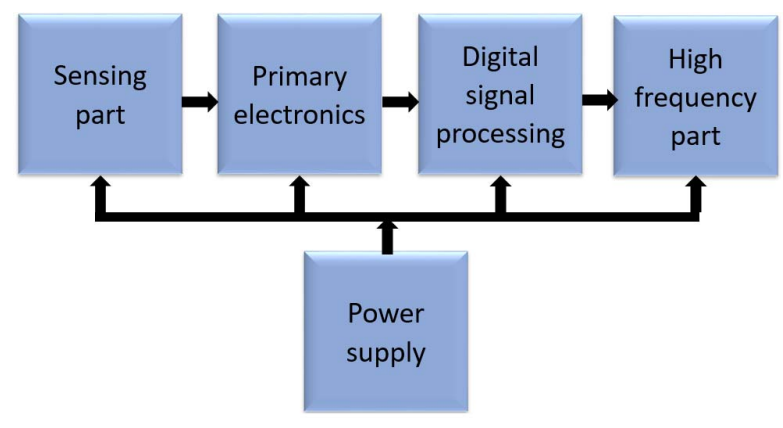

Figure 3. Sensor architecture.

The sensing part contains the proximity sensor, which indicates the presence of the sensed element. The output from this section is an analog signal. The primary electronics perform impedance matching, filtering of the signal from the sensing part and converting the analog signal into a digital signal. The digital signal is processed and evaluated in the third block. The processed signal is sent in digital form to the high-frequency part. The HF part processes the digital signal and adds the information needed for a wireless transmission. In the last step, the signal is sent via wireless medium to the receiving station. The receiving station processes the signal and sends it to the server.

\section{WIRELESS SENSOR COMPONENTS}

In selecting the appropriate hardware components for the individual blocks in the block diagram, we were started sensing part. This part is most critical in power consumption. We conducted a survey and selected steute RF IS MI8 $n b-S T$. The reason for its selection is its low power consumption and the possibility of power supply in the range of 3 to $5 \mathrm{~V}$. The primary electronics block is to ensure the supply of the whole system, filtering of unwanted voltages and adjusting the output signal from the sensing part. The primary electronics contain connectors that will allow you to connect a battery, scanner, or upload software code. The ATMEGA328P-AN processor is used for digital signal processing. The processor will communicate via USART serial communication with the highfrequency part. $i M 880 B-L o R a W A N$ is used as the RF part. The connection of these two parts can be seen in Fig. 5. This is a modular solution from company IMST. All of this will be powered by the ER26500 $3.6 \mathrm{~V}$ battery. This battery has a capacity of $8.5 \mathrm{Ah}$. We had to adjust the sensing part by impedance, then we adjusted the signal shape. We can see these adjustments in Fig. 6. The diode D1 was used to 
reduce voltage, to maintain the required limits hysteresis of the integrated circuit U1. The U1 circuit converts the analog signal to digital. After this adjustment we could connect the signal output to the digital input of the processor. If we appropriately combine the mentioned parts with a suitable connection of the primary electronics, the desired result is achieved in the form of a wireless proximity sensor. In the Fig. 4 we can see the resulting PCB, to which it is necessary to connect the proximity sensor and battery. PCB parameter are $95 \times 64 \mathrm{~mm}$.

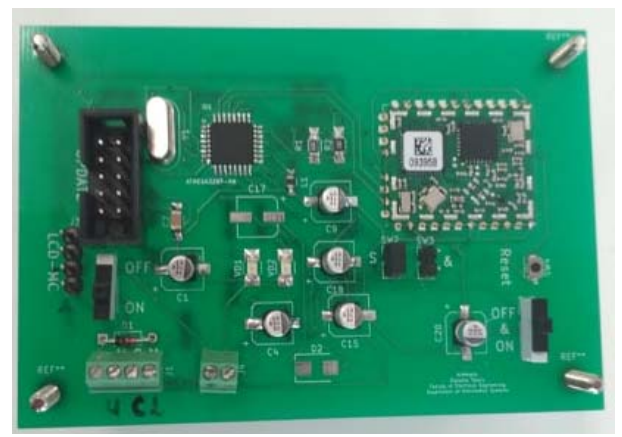

Figure 4. Wireless sensor PCB.

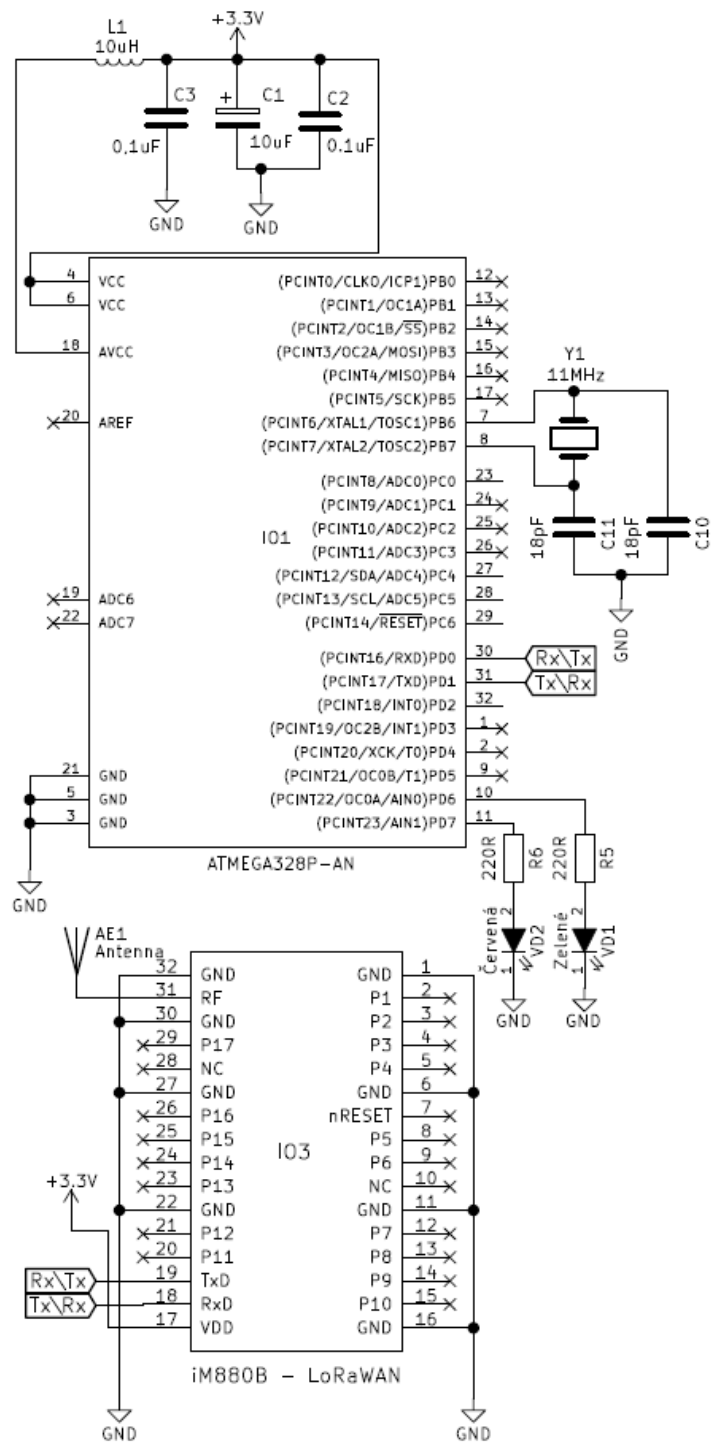

Figure. 5. Basic connection of microprocessor and RF part.

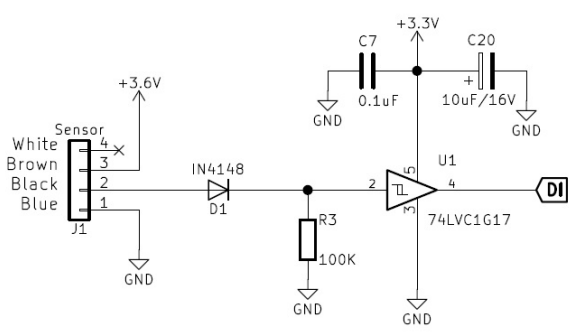

Fig. 6. Adjusting the sensor signal.

\section{FUNCTIONALITY TEST}

When the prototype was completed it was necessary to test its functionality. To simulate the real environment, a conveyor belt was used, to which metal washers were glued. The sensor was then attached to the conveyor belt. We then fastened the sensor to the conveyor belt. The result can be seen in Fig.7

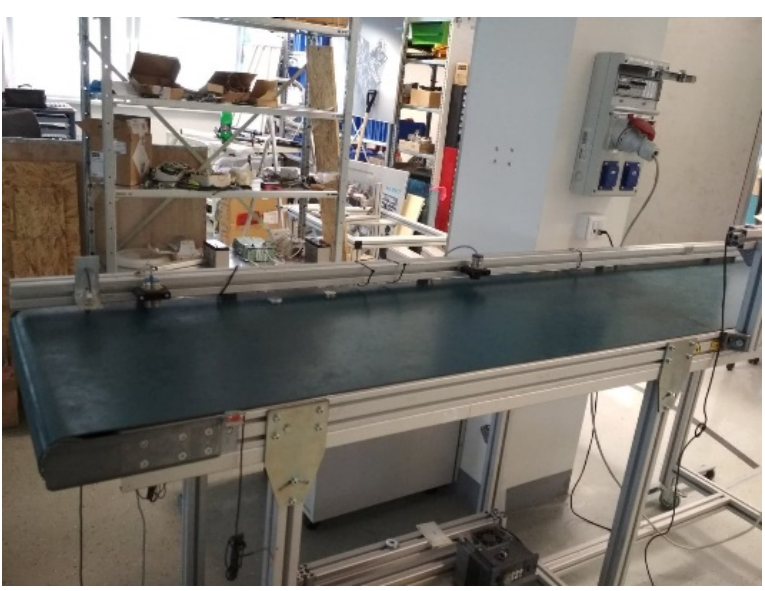

Figure 7. Conveyor belt.

One of the requirements was that communication does not have to take place in real time. Therefore, in order to comply with the duty cycle, the sending of data after the capture of ten pieces was set. This parameter will also affect the resulting power consumption. As a reference to the wireless sensor, I used the same sensor connected to an electromechanical counter that recorded each sensor switch. The results of these measurements can be seen in the Tab. 2. The SF value always started at 7. It varied during the test based on the transfer properties Setting the parameters of the radio module is a software issue that is quite complex and beyond the content of this article. You can read more about the settings in source [4]. Other measurements that needed to be done is the measurement of power consumption. This parameter is critical when deploying equipment in long-term operation. In order to achieve the best results, both the CPU power saving mode and the iM880B-LoRaWAN RF module have been set. As a result, the average power consumption was $1.82 \mathrm{~mA}$. This consumption was measured during Test 2. During the Test 2., we used a digital multimeter DM3058E, which contains statistical functions, which made the measurement easier. The results are shown on the display during the 
entire test. When transmitting peak current was 81 to $100 \mathrm{~mA}$. This depended on the individual transmission. How to achieve the best consumption will be analyzed by this resource [10]. An important part that has not yet been mentioned is the antenna. The antenna has a significant effect on the quality of the received signal. For this reason, we decided to perform a separate test, which is focused on antennas of different types. We used the RSSI (Received Signal Strength Indicator) as an antenna quality indicator. Transmission is better when RSSI is closer to zero. The output of the module iM880BLoRaWAN is designed to connect to a unipolar antenna with an impedance of $50 \Omega$. In the test, we used the purchased antennas, but we also made. The test is summarized in the table. We made the antennas A1 and A2 according to Fig. 8 [1]. They differed in the number of turns. A1 has 7 turns and A2 has 11 turns. A wire antenna is also used in the test. The wire length is $1 / 4$ wavelength. The calculation is in formula (1) where $\lambda$ is the wavelength and $f$ is the frequency of $868 \mathrm{kHz}$.

$$
\lambda=(300 / f) \times(1 / 4)=0,086[\mathrm{~m}]
$$

The test was carried out in the building at a distance of 15 meters and with direct visibility of the transmitter and receiver.

TABLE II. TEST RESULT

\begin{tabular}{|l|c|c|}
\hline Parameters & Test 1 & Test 2 \\
\hline Start test & 1.3 .2019 & 13.3 .2019 \\
& $11: 35$ & $7: 00$ \\
\hline Stop test & 1.3 .2019 & 13.3 .2019 \\
& $14: 35$ & $12: 00$ \\
\hline Number counted & 290 & 1271 \\
Wireless Sensor & & \\
\hline Number counted & 290 & 1271 \\
Ref. Sensor & & \\
\hline Number transmission & 29 & 128 \\
\hline
\end{tabular}

TABLE III. ANTENNA TEST

\begin{tabular}{|l|c|c|}
\hline Antenna & Range RSSI & Average RSSI \\
\hline A1 & $<-39,-49>$ & -46 \\
\hline A2 & $<-58,-71>$ & -64 \\
\hline Wire & $<-43,-60>$ & -50 \\
\hline None & $<-92,-108>$ & -101 \\
\hline ANT-SS900 & $<-45,-59>$ & -49 \\
\hline 8WHIP3H-HMA & $<-48,-57>$ & -50 \\
\hline
\end{tabular}

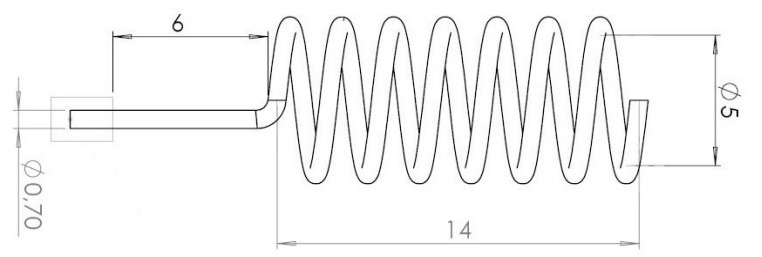

Figure 8. Mechanical dimensions of the antenna

\section{CONSOLUSION}

This sensor has been tested in several laboratory tests. From these tests, we can say that the conditions that have been established have been complied. Power consumption also achieved excellent results 1,82 mA. At current consumption and battery, the battery is expected to last 180 days.

\section{ACKNOWLEDGMENT}

This work has been supported by the Educational Grant Agency of the Slovak Republic (KEGA) Number: 016ŽU-4/2018 Modernization of teaching methods of management of industrial processes based on the concept of Industry 4.0.

\section{REFERENCES}

[1] A. Janota, V. Šimák, and J. Hrbček, "Development of a Weight-in-Motion measurement system with an optical sensor," in 17th International Conference on Transport Systems Telematics, Poland, pp. 234-247, ISSN 1865-0929, September 2017.

[2] D. Nemec et al.: Control of the mobile robot by hand movement measured by inertial sensors. Electrical Engineering, 2017, pp. 1-8, Print ISSN 0948-7921, Online ISSN 1432-0487, http://sci-hub.tw/10.1007/s00202-0170614-3

[3] A. Janota, A., V. Šimák, D. Nemec, D., J. Hrbček: Improving the precision and speed of Euler angles computing from lowcost rotation sensor data, in: Sensors - ISSN 1424-8220. Vol. 15, no. 3 (2015), online, s. 7016-7039

[4] M. Mihálik: Bezdrôtový proximitný snímač. Diplomová práca. Fakulta elektrotechniky a informačných technológii, Žilinská univerzita v Žiline, 2019, Evidenčné číslo: 28260220192010

[5] A. Zourmand, A. L. Kun Hing, C. Wai Hung and M. AbdulRehman, "Internet of Things (IoT) using LoRa technology," 2019 IEEE International Conference on Automatic Control and Intelligent Systems (I2CACIS), Selangor, Malaysia, 2019, pp. 324-330. doi: 10.1109/I2CACIS.2019.8825008

[6] A. Trunov and A. Malcheniuk, "The Mesh Technology for Improving of End Nodes Updating for Lorawan Network," 2018 IEEE 13th International Scientific and Technical Conference on Computer Sciences and Information Technologies (CSIT), Lviv, 2018, pp. 164-167. doi: 10.1109/STC-CSIT.2018.8526649

[7] N. Benkahla, B. Belgacem and M. Frikha, "Security analysis in Enhanced LoRaWAN duty cycle," 2018 Seventh International Conference on Communications and Networking (ComNet), Hammamet, Tunisia, 2018, pp. 1-7. doi: 10.1109/COMNET.2018.8622296

[8] B. Oniga, V. Dadarlat, E. De Poorter and A. Munteanu, "A secure LoRaWAN sensor network architecture," 2017 IEEE SENSORS, Glasgow, 2017, pp. 1-3. doi: 10.1109/ICSENS.2017.8233990

[9] P. Peniak and E. Bubeníková, "Validation of IoT secure communication gateway for constrained devices," 2019 International Conference on Applied Electronics (AE), Pilsen, Czech Republic, 2019, pp. 1-5., DOI: 10.23919/AE.2019.8866990

[10] D. Tokmakov, S. Asenov and S. Dimitrov, "Research and development of ultra-low power LoraWan sensor node," 2019 IEEE XXVIII International Scientific Conference Electronics (ET), Sozopol, Bulgaria, 2019, pp. 1-4. doi: 10.1109/ET.2019.8878674

[11] ChirpStack, "Open-source LoRaWAN Network server stack" Online : www.chirpstack.io 